\title{
Epicardial excitation of the atria in a patient with atrial flutter
}

\author{
Hein J. J. Wellens, Michiel J. Janse, Rudolf T. van Dam, and Dirk Durrer \\ From the University Department of Cardiology and Clinical Physiology, \\ Wilhelmina Gasthuis, Amsterdam, The Netherlands
}

Atrial epicardial excitation mapping was performed during open heart operation in a patient with atrial flutter and rheumatic mitral and aortic valve disease. Though it was impossible to study completely the epicardial excitation of both atria, our results do exclude a circus movement involving a large part of the atria. The two possible mechanisms responsible for the atrial flutter - in our patient were: (I) an ectopic focus located low in the atrium; and (2) a reciprocal rhythm situated in a small area low in the atrium or upper part of the AV junction.

There is no unanimous opinion on the mechanism of atrial flutter in man.

The two most favoured theories are: a circus movement running over the atrium, or a rapidly firing ectopic atrial focus. Recently we had the opportunity to study atrial epicardial excitation directly in the human heart as exposed during cardiac surgery.

\section{Report of a case}

The patient was a 45-year-old man, who was admitted to our department for the evaluation of rheumatic aortic regurgitation and mitral stenosis.

At the age of 17 he suffered from an attack of acute rheumatic fever. Seven years before admission paroxysms of atrial flutter were registered; 3 years later the atrial flutter became permanent (Fig. I).

- Received 6 July 1970.
Examination at our department, including right and left heart catheterization, revealed the presence of severe aortic regurgitation and moderately severe mitral stenosis. In view of his functional classification (grade $3 / 4$ of the New York Heart Association), it was decided to replace his aortic valve by a Starr-Edwards prosthesis and to perform an open mitral commissurotomy.

Before this procedure it was planned to study epicardial excitation of the atria.

Exploration of epicardial excitation The methods of recording epicardial leads have been described previously (Durrer et al., 1961). Briefly, unipolar and bipolar complexes were recorded using a hand-held electrode carrying 2 silver electrode terminals of $0.1 \mathrm{~mm}$. diameter, the interelectrode distance being $\mathrm{I} \mathrm{mm}$. The locations of the recording sites were entered on carefully drawn maps of the atrial surface. The signals were recorded simultaneously with a constant bipolar

FIG. I Lead III (shown here at greater magnification) revealing the presence of atrial flutter (flutter cycle length 235 msec.).

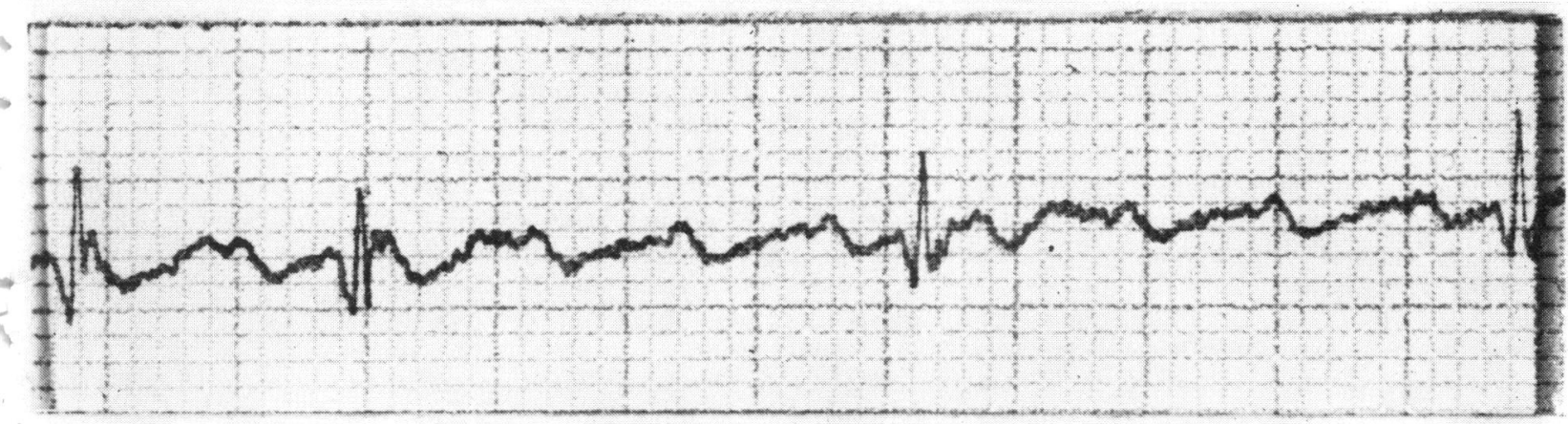


reference tracing taken from a bipolar disc electrode sewn upon the right atrial appendage, and the standard lead II electrocardiogram. All complexes were recorded from a high fidelity 4-channel oscilloscope on $36 \mathrm{~mm}$. film at a speed of $2 \frac{1}{2} \mathrm{in}$./ sec. Time pips occurred every $100 \mathrm{msec}$. For the purpose of time measurements the recordings were enlarged to such an extent that I $\mathrm{mm}$. corresponded to I msec.

The rapid part of the intrinsic deflection signalled the arrival of the excitatory wave in the epicardial layer in contact with the exploring electrode (Durrer and van der Tweel, r957).

\section{Results}

The number of atrial epicardial complexes that could be recorded was limited by: (I) the presence of fat on the posterolateral aspect of the right atrium preventing the registration of atrial complexes showing a rapid intrinsic deflection; and (2) the fact that the left atrium could only be reached by lifting the heart out of the thorax. This was immediately followed by a severe drop in blood pressure. Therefore only a limited amount of left atrial complexes could be recorded. Because of this it was impossible to map completely the excitation of both atria.

Our results from the 37 epicardial complexes that could be recorded indicate that the earliest point of atrial epicardial excitation was at the medial surface of the right atrial appendage, close to the aortic root, where the appendage passes into the body of the right atrium. Subsequent activation times with their corresponding epicardial complexes are shown in Fig. 2, 3, and 4. The latest point of excitation was found on the left atrial appendage (192 msec. at a flutter interval of 235 msec.). As shown in Fig. 3, excitation of the left atrium close to the aorta occurred at 142 msec. It is important to realize that the left atrium was much enlarged and was hanging over the right atrium. Though this point was situated spatially closely to the earliest point of right atrial activation, they were separated by a large amount of atrial tissue.

\section{Discussion}

In 192 I Lewis, Drury, and Iliescu introduced the concept of a circus movement as the actual mechanism of atrial flutter in man. Since then other theories have been offered. From these, the one that considers atrial flutter in man as caused by a rapidly firing ectopic focus has received most support.

Both the circus movement and the ectopic focus theory have enthusiastic advocates. This is well illustrated by two recent articles that review extensively experiments, theories,

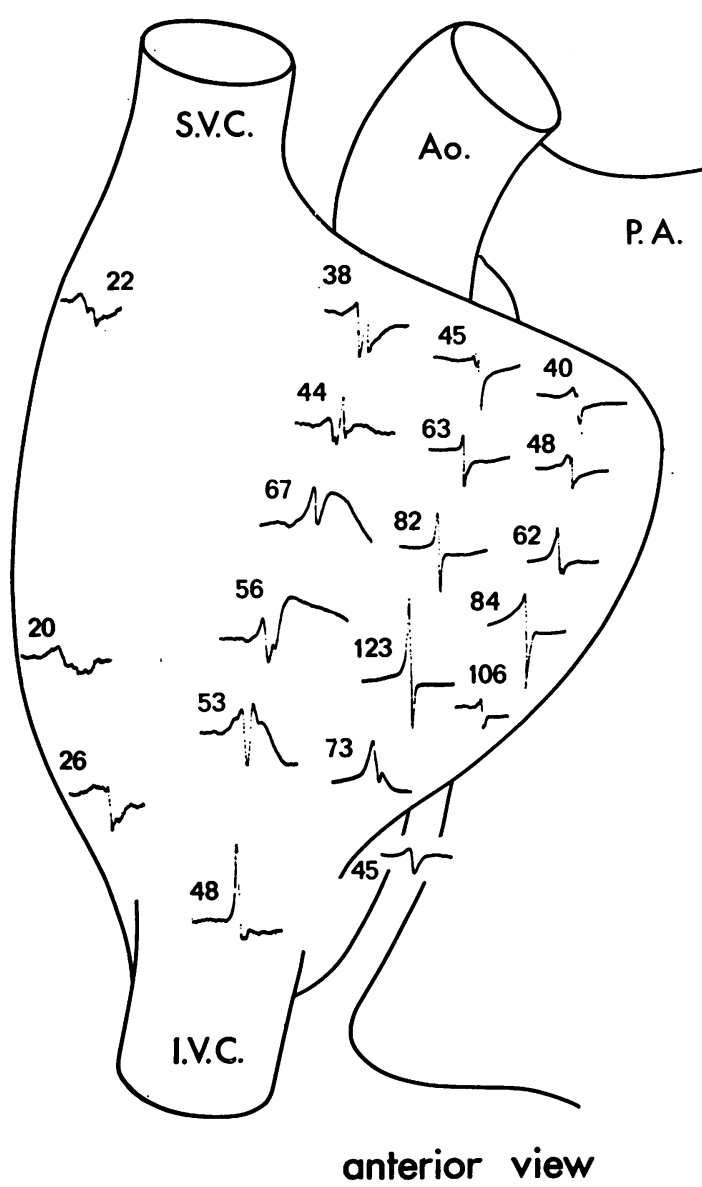

FIG. 2 Epicardial complexes and activation times as recorded from the anterior side of the right atrium, right atrial appendage, and left atrial appendage.

and knowledge on this subject. Rytand (1966) closes his article with the sentence: 'The circus movement hypothesis appears to account for the mechanism of pure atrial flutter of the classical type'. Scherf (I966) summarizes: 'On the basis of the available data it must be concluded that experimental flutter can be caused by the rapid formation of impulses, whereas the theory of re-entry still requires proof. There is no definite proof for either mechanism in clinical flutter'.

Supporters of both theories have used intracavitary and intra-oesophageal leads to prove their point. An ectopic focus was favoured by Kossmann et al. (1950), Coelho, da Fonseca, and Nunes (1950), Prinzmetal et al. (1952), and Latour and Puech (1957), a circus movement by Steinberg et al. (1950) and Rytand (1966).

Wenger and Hofmann-Credner (I952) and Kishon and Smith (1969) described two 
FIG. 3 Epicardial complexes and activation times as recorded on the inner surface of the right atrial appendage showing the earliest point of epicardial breakthrough close to the aortic root.

groups of patients, one where a circus movement was the most likely mechanism, and one where an ectopic focus seemed responsible for the atrial flutter. Katz and Pick (I960) also believe that different types of atrial flutter exist and that both mechanisms can be present. Intra-atrial leads, even multiple ones taken simultaneously with standard leads, have in our experience not been very helpful in solving the problem. Our difficulty was the exact location of the electrode catheter as far as its distance to the atrial septum and free atrial wall was concerned.

We therefore welcomed the opportunity to study directly atrial activation during atrial flutter, when our patient was operated on. To our knowledge this has been performed only once before, by Hurley and Shumway (as -reported by Rytand, 1966). They concluded from their activation times that a circus movement was present in their patient. Evidence of atrial excitation during a large part of or almost the entire flutter cycle has been regarded as suggestive of the existence of a circus movement. One should realize, howrever, that: (a) there is slowing of impulse propagation through the atrial wall at rapid atrial 'rates; (b) pathology of the atrial wall is frequently present in patients with atrial flutter.

In our patient who suffered from rheumatic heart disease atrial excitation covered at least $192 \mathrm{msec}$. of the flutter cycle length of 235 msec. As shown in Fig. 2-5, the location of the earliest and latest point of atrial epicardial excitation does exclude the presence of a circus movement, covering a large part of the atria.

Our findings are compatible with an ectopic focus as the causal mechanism for the atrial flutter in our patient. The earliest point of right atrial epicardial excitation was found at the medial surface of the right auricle, close to the aortic root, where it merges with the body of the right atrium.

This area corresponds with the earliest site of epicardial breakthrough found by Goodnan, van der Steen, and van Dam (I97I) in ;ome of their dogs during stimulation near he coronary sinus. They postulated that this

YG. 4 Epicardial complexes and activation imes as recorded from the posterior side of the ight and left atrium.

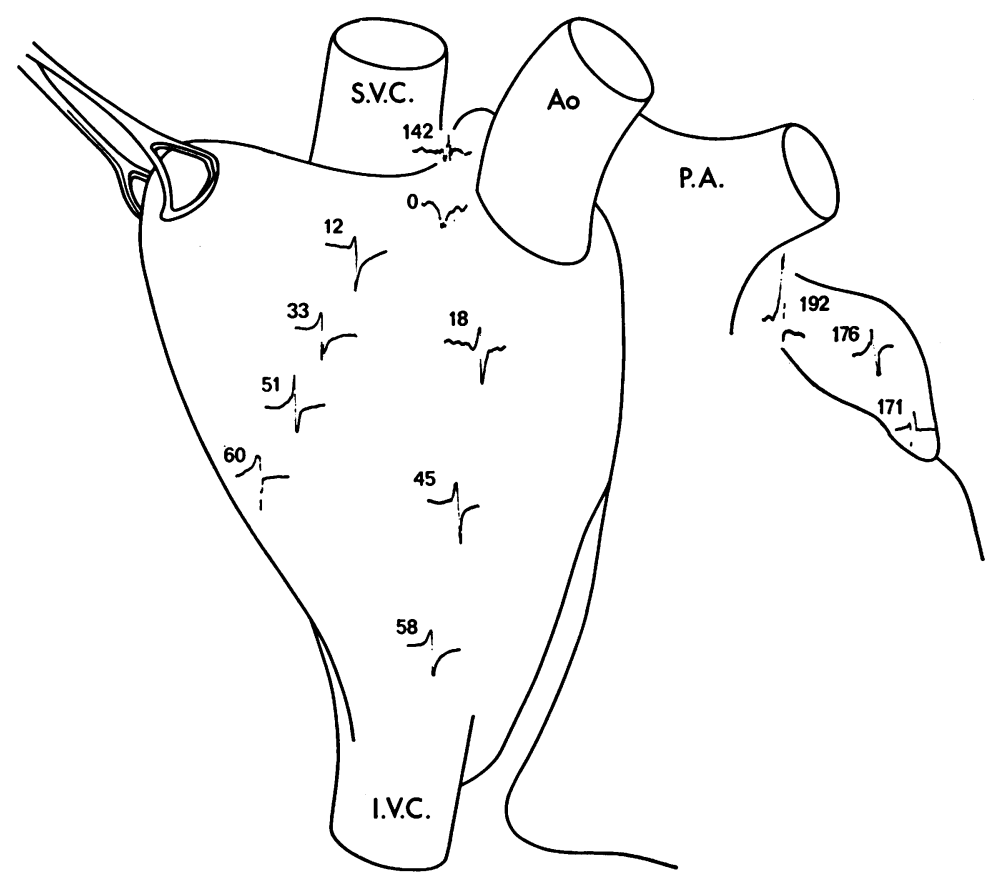

medial surface right auricle

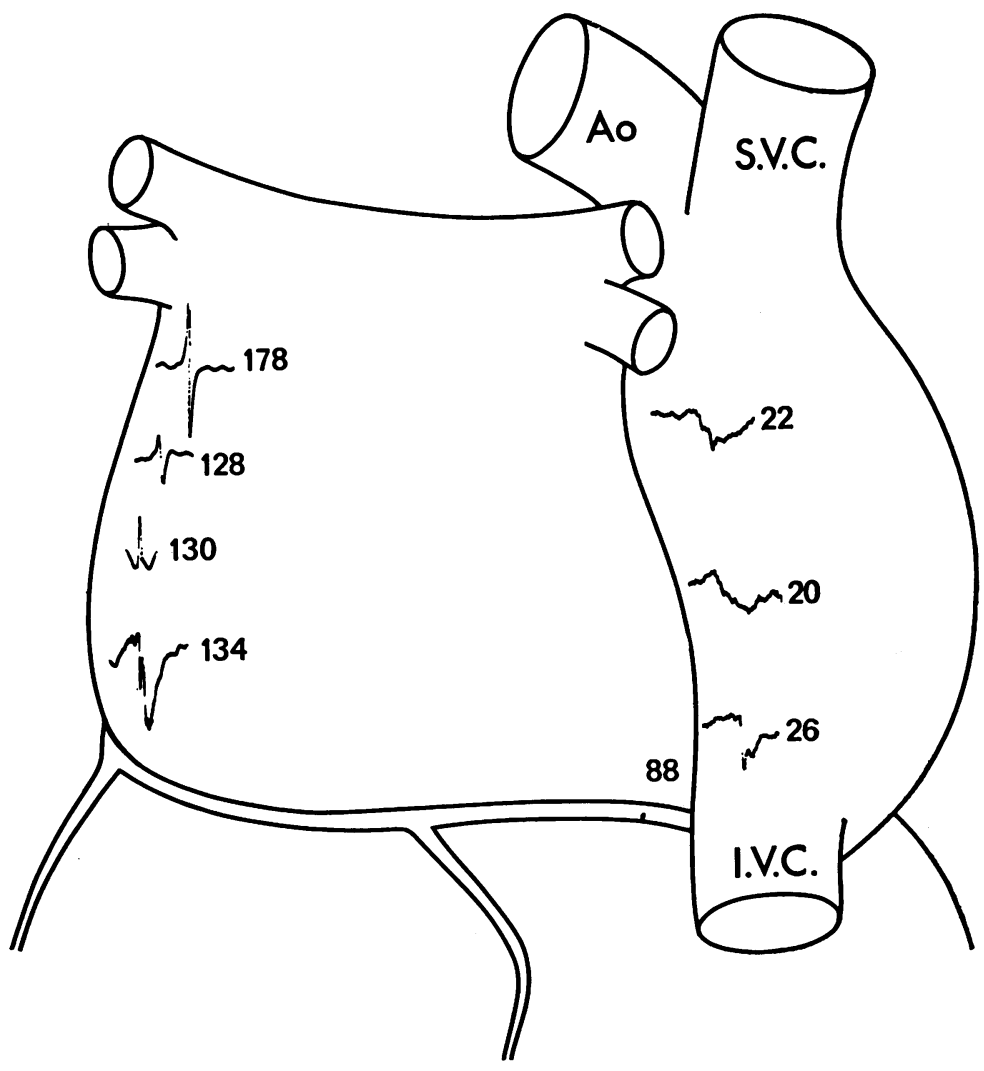

posterior view 
could have been the result of rapid retrograde conduction, along the anterior internodal tract (James, 1963; Merideth and Titus, 1968) for a short distance, followed by more or less radial epicardial spread. A similar activation pattern was seen during retrograde atrial excitation in the isolated revived human heart (Durrer et al., 1970).

Recently the close resemblance was reported between a unifocal tachycardia produced by rapid pacing from the coronary sinus and atrial flutter (Rosen, Lau, and Damato, 1969). The outcome of our epicardial excitation mapping and the studies enumerated above have led us to suggest a sequence of atrial excitation in our patient, as shown in Fig. 5. Though we do not know exactly the site of origin of the atrial flutter in our patient, we feel that a location low in the atrium close to the AV node is the most likely one. The early epicardial breakthrough high at the anterior side of the right atrium can be the result of rapid retrograde conduction along the anterior internodal tract. Thereafter more or less radial epicardial spread occurred.

The activation times of the lower and lateral right atrium and of the (much enlarged) left atrium can be accounted for by assuming radial spread of excitation from the site of origin of the impulse low in the atrium. The limited amount of data on left atrial activation does not allow conclusions as to the presence of preferential conduction pathways to the left atrium. Unfortunately we are not informed about the mechanism at the site of origin of the atrial flutter in our patient. It is impossible to decide between: (I) an ectopic focus located low in the atrium, or (2) a reciprocal rhythm situated in a small area low in the atrium or in a longitudinally dissociated upper part of the AV junction. It is obvious that the ideas enumerated above can only be applied to the patient studied. We certainly do not want to suggest that this activation pattern is present in all patients with atrial flutter.

We greatly appreciate the help of Professor N. G. Meijne, who made this study possible.

\section{References}

Coelho, E., da Fonseca, J. M., and Nunes, A. (1950). Les altérations du potentiel intra-auriculaire. Cardiologia, 17, 346.

Durrer, D., Büller, J., Graaff, P., Lo, G. I., and Meyler, F. L. (196I). Epicardial excitation pattern as observed in the isolated revived and perfused fetal human heart. Circulation Research, 9, 29.

-, van Dam, R. T., Freud, G. E., Janse, M. J., Meijler, F. L., and Arzbaecher, R. C. (1970). Total excitation of the isolated human heart. Circulation, 41, 899.

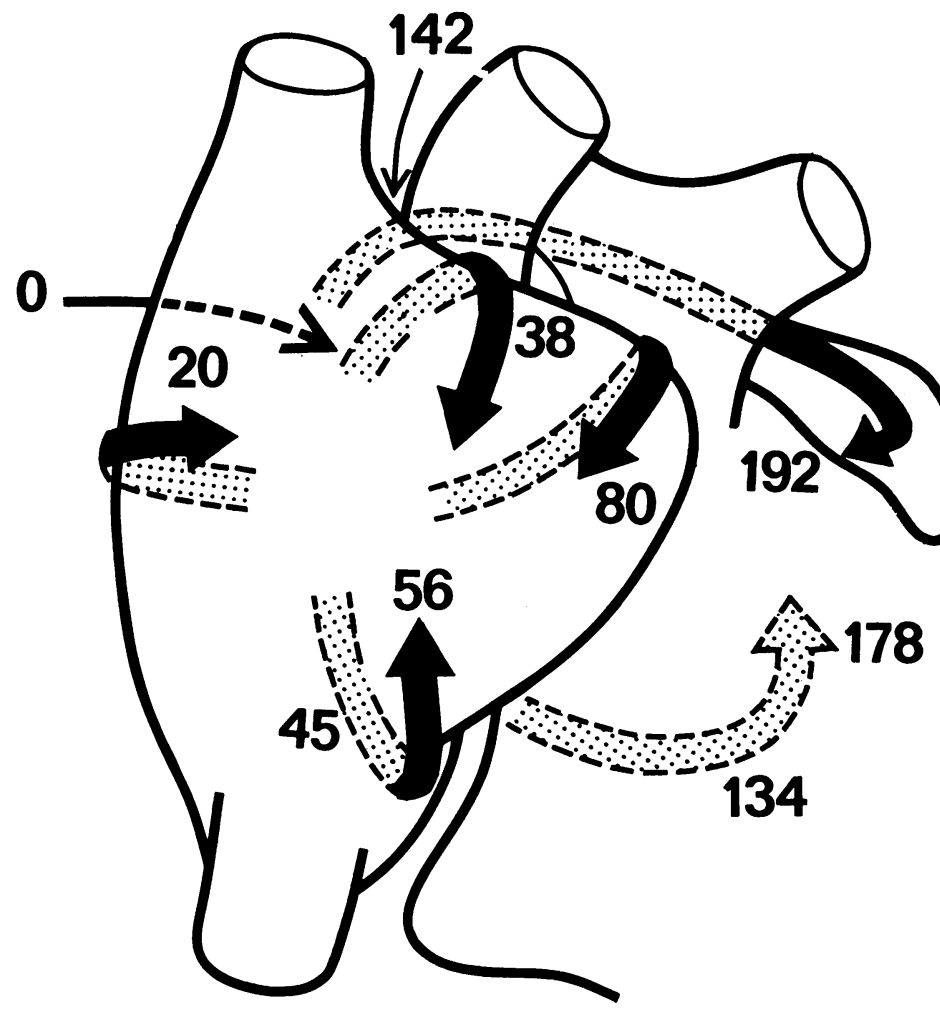

FIG. 5 Scheme showing the suggested spread of atrial excitation in our patient during atrial flutter (flutter cycle length $235 \mathrm{msec}$.).

-, and van der Tweel, L. H. (1957). Excitation of the left ventricular wall of the dog and goat. Annals of the New York Academy of Sciences, 65, 779.

Goodman, D., van der Steen, A. B. M., and van Dam, R. T. (197I). Endocardial and epicardial activation of the canine right atrium. American fournal of Physiology. In the press.

James, T. N. (1963). The connecting pathways between the sinus node and AV node and between the right and the left atrium in the human heart. American Heart fournal, 66, 498.

Katz, L. N., and Pick, A. (1960). Current status of theories of mechanisms of atrial tachycardias, flutter and fibrillation. Progress in Cardiovascular Disease, 2, 650.

Kishon, Y., and Smith, R. E. (1969). Studies in human atrial flutter with the use of proximity electrodes. Circulation, 40, 513.

Kossmann, C. E., Rader, B., Berger, A., Brumlick, J., and Briller, S. A. (1950). Electrograms in atrial flutter, atrial tachycardia, and atrial fibrillation. In $I^{\text {er }}$ Congrès Mondiale de Cardiologie, Paris, 1950. Communications: Résumés, p. 318.

Latour, H., and Puech, P. (1957). Electrocardiographie Endocavitaire. Masson, Paris.

Lewis, T., Drury, A. N., and Iliescu, C. C. (I92I). A demonstration of circus movement in clinical flutter of the auricles. Heart, 8, 34I.

Merideth, J., and Titus, J. L. (I968). The anatomic atrial connections between sinus and AV node. Circulation, 37, 566. 
Prinzmetal, M., Corday, E., Brill, I. C., Oblath, R. W., and Kruger, H. E. (1952). The Auricular Arrhythmias. Charles C. Thomas, Springfield, Illinois.

Rosen, K. M., Lau, S. H., and Damato, A. N. (1969). Simulation of atrial flutter by rapid coronary sinus pacing. American Heart fournal, 78, 635.

Rytand, D. A. (1966). The circus movement (entrapped circuit wave) hypothesis and atrial flutter. Annals of Internal Medicine, 65, 125.

Scherf, D. (1966). The mechanism of flutter and fibrillation. American Heart fournal, 71, 273.
Steinberg, M. F., Grishman, A., Kroop, I. G., and Jaffe, H. L. (1950). A study of circus movement in human atrial flutter by means of intracardiac, esophageal and vectorcardiography. In I ${ }^{\text {er }}$ Congrès Mondiale de Cardiologie, Paris, 1950. Communications: Résumés, p. 54

Wenger, R., and Hofmann-Credner, D. (1952). Observations on the atria of the human heart by direct and semidirect electrocardiography. Circulation, 5,870 . 